\title{
Research Paper \\ Effect of Eight Weeks of Concurrent Training on Liver Enzymes, Lipid Profile, and Insulin Resistance Among Overweight Male Children
}

\author{
Shahrirar Khajeh Salehani ${ }^{1} \odot{ }^{*}$ Rostam Alizadeh $^{2}$
}

1. Department of Sports Science, School of Humanities, Islamic azad University, North Tehran Branch, Tehran, Iran.

2. Department of Sports Science, School of Literature and Humanities, Ilam University, Ilam, Iran.

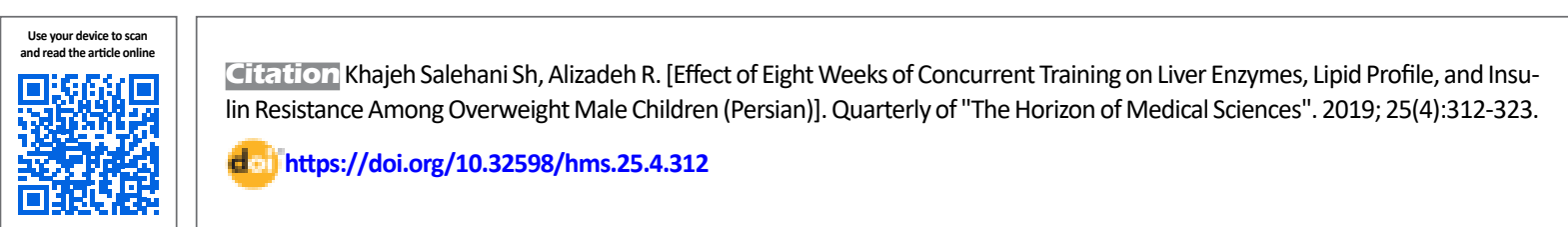

Received: 12 Feb 2019 Accepted: 03 Aug 2019 Available Online: 01 Oct 2019

Key words: Parallel exercise training, Lipid profile, Alanine transaminase, Weight loss

\section{ABSTRACT}

Aims The aim of this study was to investigate the effect of eight weeks of combined exercise training (aerobic-resistance) on liver enzymes, lipid profile, and insulin resistance among overweight boys. Methods \& Materials Thirty-two healthy overweight boys voluntarily agreed to take part in the study following the related announcement. The overweight children were randomly divided into control $(n=16)$ and experimental groups $(n=16)$. The latter group performed Concurrent training for eight weeks, three sessions per week, on non-consecutive days. In the same way as the experimental group, both before and after the eight weeks of training. In similar conditions, measurement were performed for both experimental and control groups in two stages (i.e. pre-test and after eight weeks of training).

Findings The results of comparing pre- and post-exercise changes in the values for glucose, insulin, ALT, AST, and insulin resistance for the combined exercise training group showed a significant decrease when compared with those for the control group $(P<0.05)$; however, the LDL and HDL values did not change significantly across groups ( $\mathrm{P}>0.05)$.

Conclusion In general, the results of this study showed that combined exercises (aerobic-resistance), by reducing the rest levels of liver enzymes, glucose, insulin, insulin resistance, and body composition indexes among 11- to 13-year-old boys, tended to be effective in decreasing the risks of being overweight and in preventing such diseases as obesity, diabetes, and non-alcoholic fatty liver disease.

\section{Extended Abstract}

\section{Introduction}

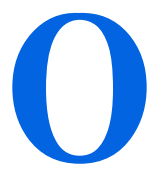

verweight in children is associated with increased mortality from cardiovascular disease in adulthood [1]. Studies that have examined the effects of physical activity and exercise on body weight and body composition have found similar results, and most have shown that exercise programs have positive effects on them. Most of these studies have focused on aerobic exercise (for example, running and cycling) and their main goal has been to increase calorie intake [1] Resistance training also has an important role in increasing muscle mass and improving physical fitness [6]. Woo et al. in a study using a combination of aerobic and resistance exercises showed that these exercises in overweight and obese children aged 9-12 years can reduce waist-to-hip ratio, but the change in body mass index,

\section{* Corresponding Author:}

Rostam Alizadeh, PhD.

Address: Department of Sports Science, School of Literature and Humanities, Ilam University, Ilam, Iran.

Tel: +98 (912) 6961587

E-mail: r.alizadeh@ilam.ac.ir 
body fat or lean body mass was not very noticeable [4]. Moreover, during one year of exercise, a decrease in body fat was observed in the exercise group who continued their exercise. However, despite the decrease in fat percentage, their weight did not change, which indicates an increase in lean body mass [4]. Combined resistance and endurance training is used as a training method by athletes and non-athletes. Some research has shown that these exercises can reduce body fat mass. The aim of this study was to assess the effect of 8-week concurrent training on liver enzyme levels, lipid profile and insulin resistance among overweight male children.

\section{Method}

This is a quasi-experimental study conducted in Tehran in 2018. Healthy overweight boys between the ages of 11 and 13 years were invited to participate in the study voluntarily and consciously at school. Prior to the study, the research method was explained to them and then their parents signed an inform consent form. Using Cochran formula, the sample size of 32 was determined and samples were selected based on the inclusion criteria and convenience sampling technique. They were divided into two groups of exercise and control.

\section{Results}

Participants had body mass index of 23 . Their mean age was $11.85 \pm 0.7$ years in the exercise group and $11.75 \pm 0.8$ years in the control group, but the difference was not significant $(\mathrm{P}=0.64)$. Their mean body height was $150 \pm 3 \mathrm{~cm}$ in the exercise group and $149 \pm 3 \mathrm{~cm}$ in the control group. Weight, body mass index, fat percentage, waist-to-hip ratio and maximum oxygen uptake of the two groups before and after exercise are presented in Table 1. The results showed that changes in body weight $\left(\mathrm{t}_{18.4}=6.01\right.$, $\mathrm{P}<0.001)$, body mass index $\left(\mathrm{t}_{18 / 62}=5.94, \mathrm{P}<0.001\right)$, fat percentage $\left(\mathrm{t}_{18.62}=5.53, \mathrm{P}=0.007\right)$ and maximum oxygen uptake $\left(\mathrm{t}_{15.76}=3.37, \mathrm{P}<0.05\right)$ in the exercise group was significantly higher than in the control group.

\section{Conclusion}

Combined exercises (aerobic and resistance training), by reducing resting levels of liver enzymes, glucose, insulin, insulin resistance and body composition indices, in 11-13 year-old boys can be an effective strategy to reduce the risks of overweight and prevent some diseases such as diabetes, obesity, and non-alcoholic fatty liver disease. It should be emphasized that, in spite of the significant reduction in many risk indicators, more time should be devoted to performing regular exercises, and a fundamental change should be made in lifestyle of children, especially in terms of physical activity. In addition, more research is needed on the potential role of a variety of exercise modalities in the prevention and treatment of many metabolic diseases, especially diabetes and non-alcoholic fatty liver, which are prevalent among children.

\section{Discussion}

The present study had some limitations. For example, it was not possible for us to have an aerobic and resistance groups alone. In general, because this type of exercise used in our study was able to simultaneously increase muscle mass reduce fat percentage (increased muscle mass leads to increased basal metabolic rate, which greatly helps to reduce fat). Also, because of the specificity of the effects of exercise, combining both endurance and resistance training is recommended for optimal physical function and health [20]. Resistance and endurance training are consistent to the extent that the number of endurance training sessions is not reduced.

\section{Ethical Considerations}

\section{Compliance with ethical guidelines}

This study has obtained its ethical approval from the Research Ethics Committee of Ilam University of Medical Sciences (Code: IR.MEDILAM.REC.1397.030). After explaining the study objectives and method, a written informed consent was obtained from the participants and their parents. They were assured of the confidentiality of their information. They were free to leave the study at any time.

\section{Funding}

This study received no financial support from any organization.

\section{Authors' contributions}

Conceptualization, methodology and initial draft preparation by Shahriar Khajeh Salehani; Conceptualization, data analysis, editing, final draft preparation and supervision by Rostam Alizadeh.

\section{Conflicts of interest}

The authors declared no conflict of interest. 
This Page Intentionally Left Blank 


\title{
تأثير هشت هفته تمرين تركيبى بر سطح آنزيمهاى كبدى، نيهرخ ليبيدى و مقاومت به انسولين در

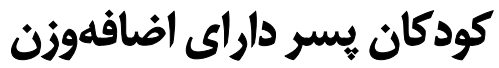

\author{
شهريار خواجه صالحانى' • •رستم علىزاده' \\ ا. كروه فيزيولورى ورزشي، دانشكده علوم انسانى، دانشعاه آزاد اسلامى واحد تهران شمال، تهران، ايران.

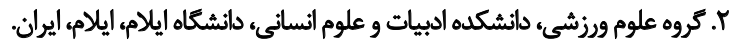

\begin{abstract}
حكن

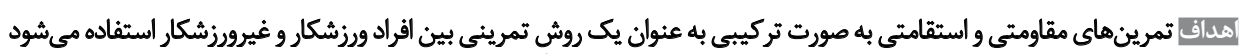

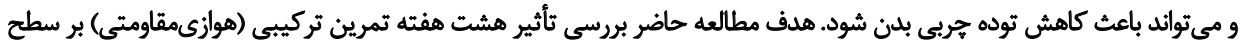

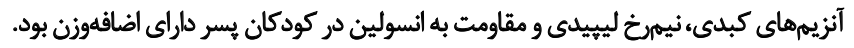

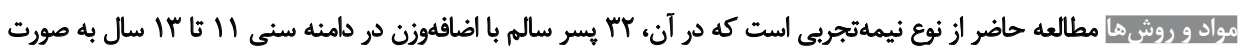

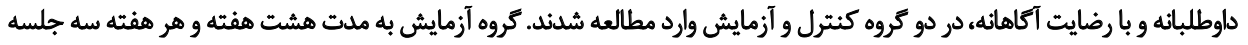

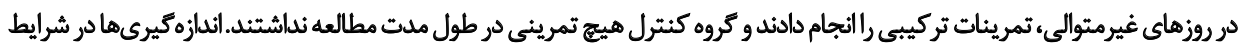

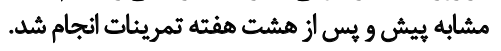

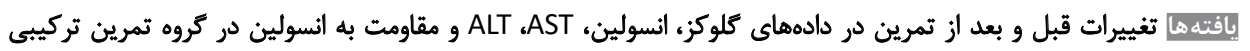

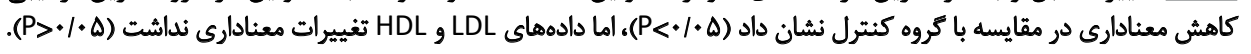

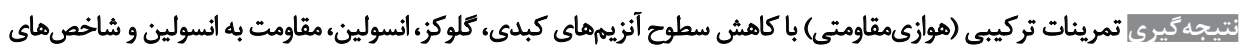

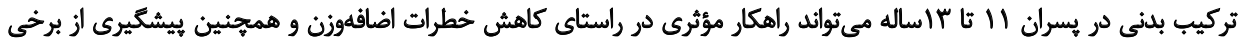

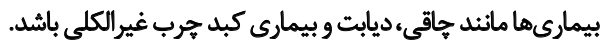

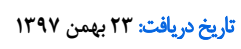

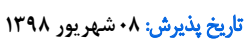

تاريخ انتشار: 9. مهر

كليدوازوهها:

تمرين موازي، نيهرخ

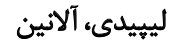
ترانسفراز، كاهش وزن

[1]. در بررسى تأثير تمرين هوازى بر اجزاى مقاومت به انسولين

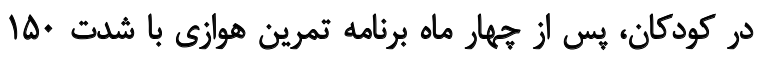

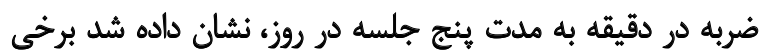

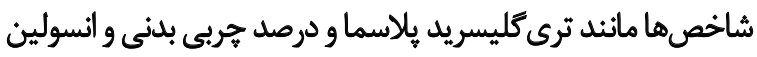

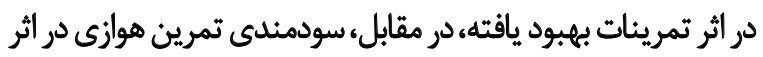

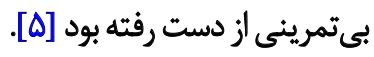

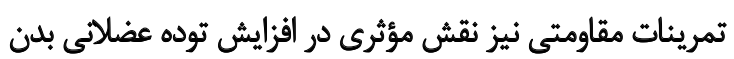

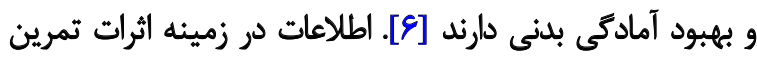

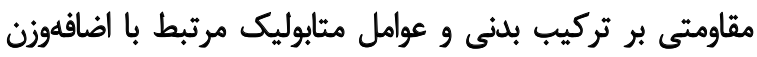

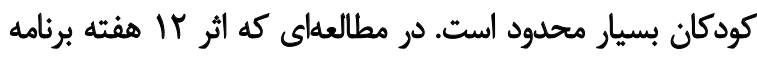

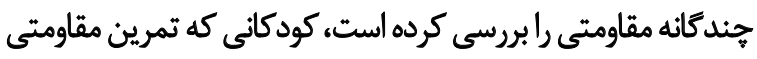

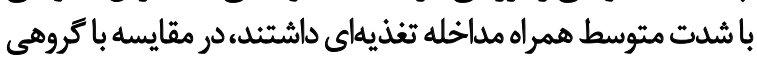

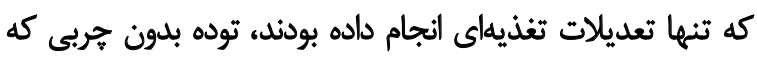

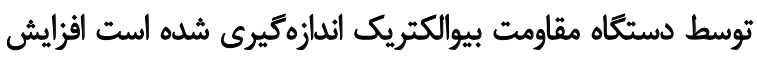

بيشترى نشان داده بود [V] توسوان.

اضافهوزن در كودكان با افزايش مركىومير ناشى از بيمارى

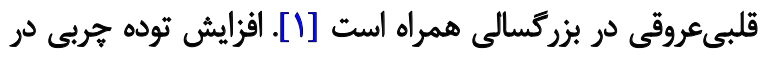

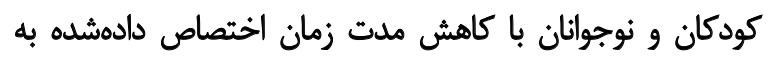

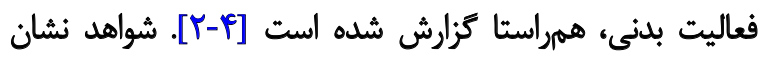

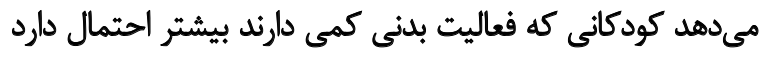

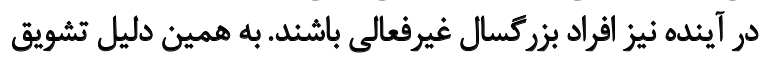

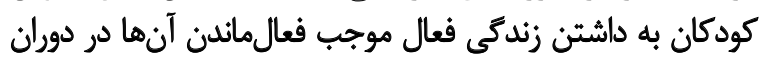

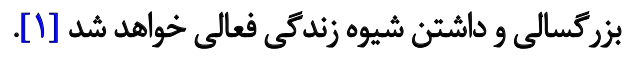

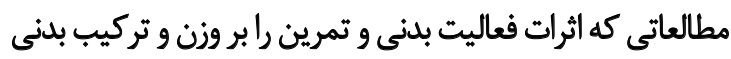

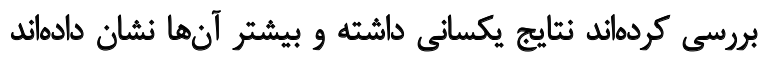

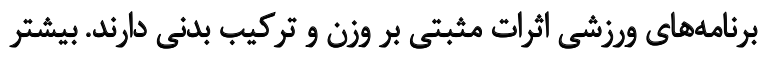

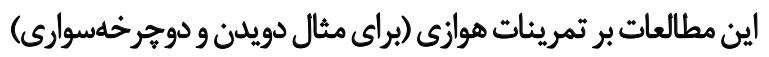

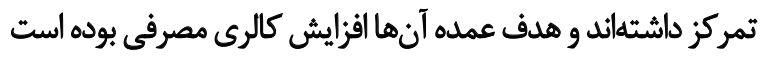

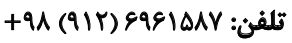
r.alizadeh@ilam.ac.ir يست الكترونيكى 
سالم داراى اضافهوزن در دامنه سنى 1 التا تا سال از طريق فراخون

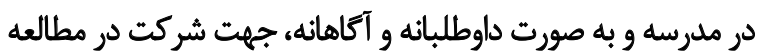

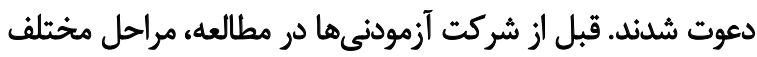

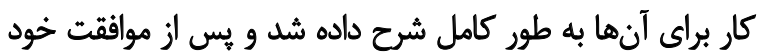

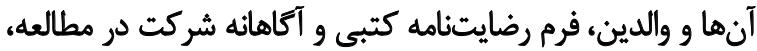

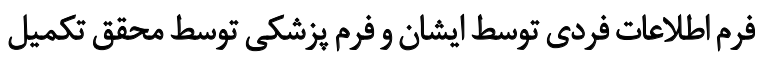

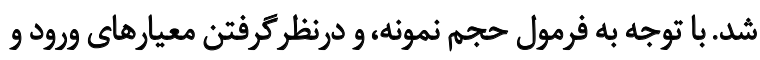

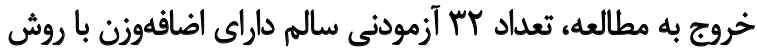
نمونهكيرى دردسترس جهت مطالعه، انتخاب شدند.

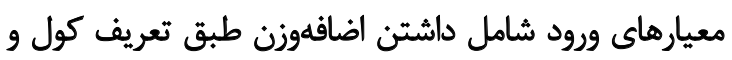

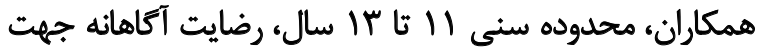
شركت در مطالعه، عدم ابتلا به هركونه بيمارئه

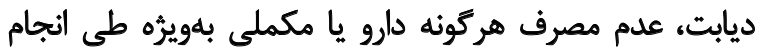

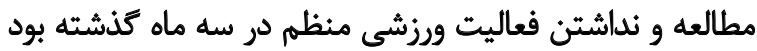

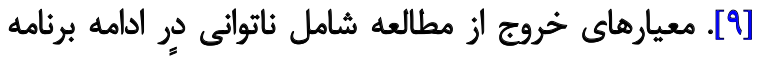

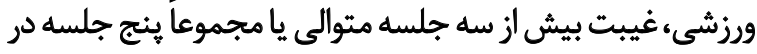

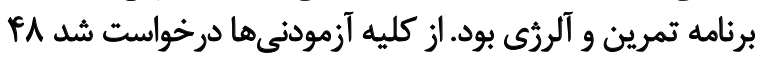

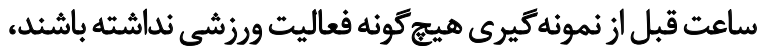

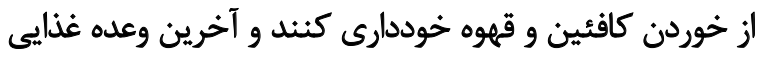

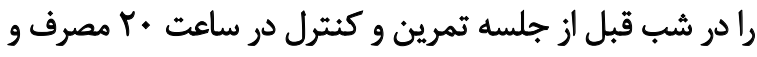
صبح در حالت ناشتا به آزمايشكاه مراجعه كنيند.

\section{كروههاي موردمطالعه و يوروتكل تمرينات}

در ابتداي مطالعه و قبل از شروع تمرينات، سنجش تركيب

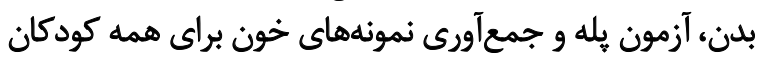

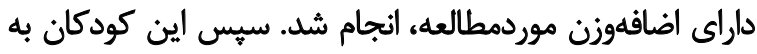

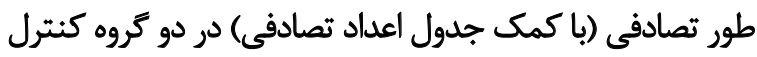

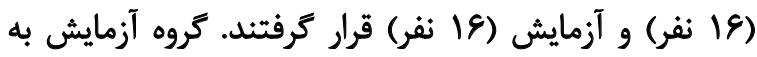

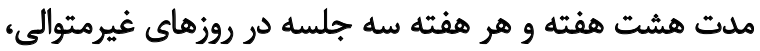

وو و همكاران نيز در بررسى تركيبى از تمرينات هوازى و مقاومتى

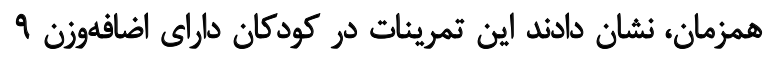

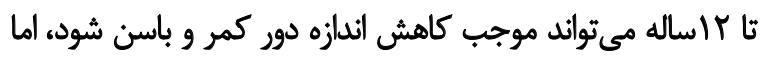

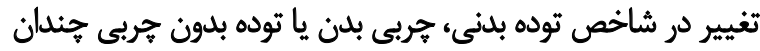

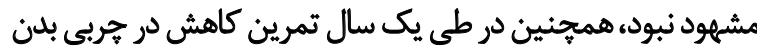

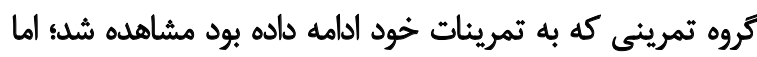

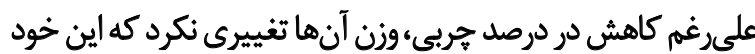

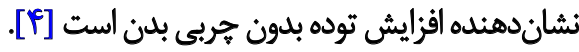

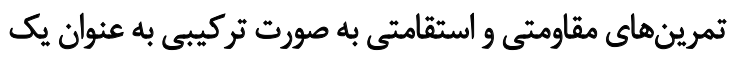

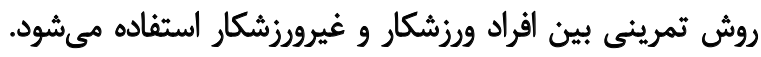

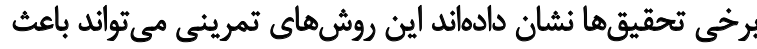

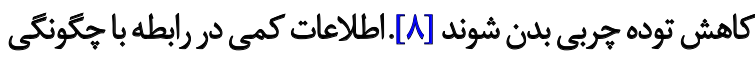

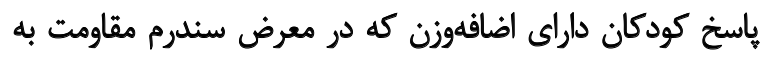

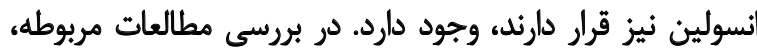

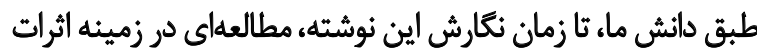

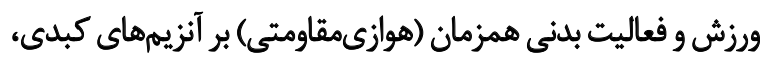

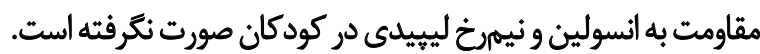

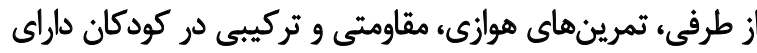

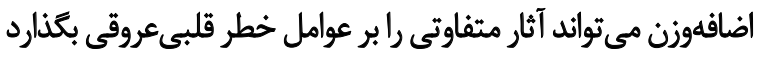

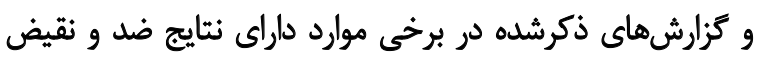

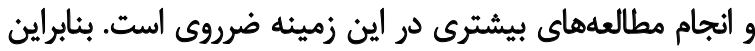

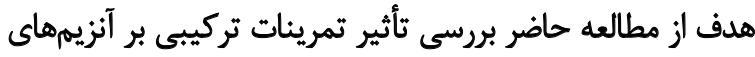

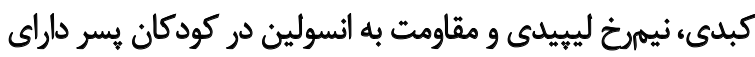

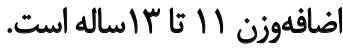

\section{مواد وروش لها}

مطالعه حاضر از نوع نيمهتجربى و كاربردى است كه بـ إس از تصويب

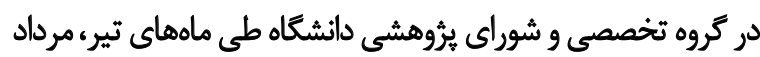

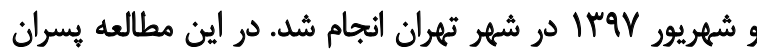

جدول ا. يروتكل تمرينات ورزشي همزمان (هوازىمقاومتى) براى كروه آزمايش (19 هير سالهم با اضافهوزن)

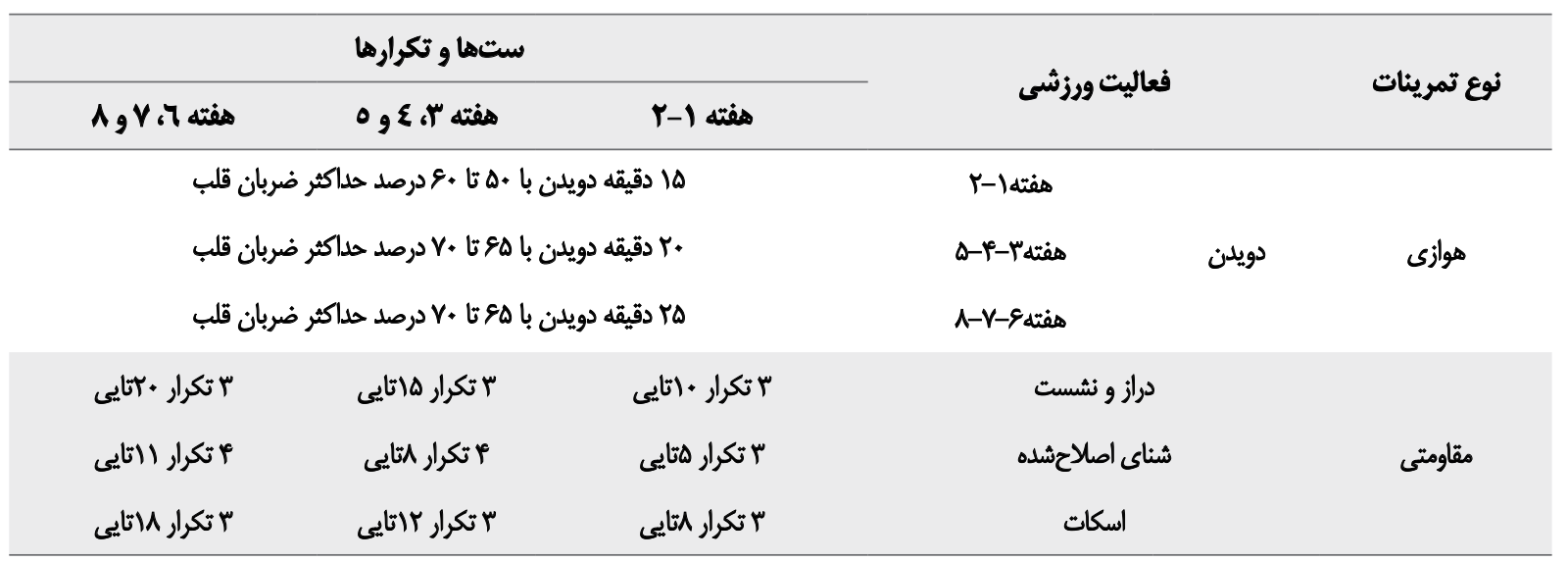

نें 
با استفاده از روش الايزاى ساندويجى و كيت انسولين (كميانى

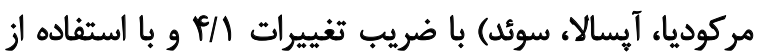

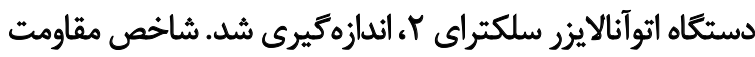

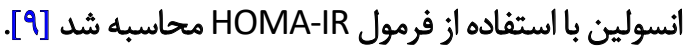

\section{تجيوزيه و تقايليل آمارى}

كليه دادهها با استفاده از نسخه 19 نرمافزار آمارى SPSS تجزيه

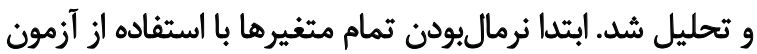

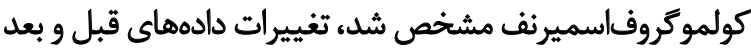

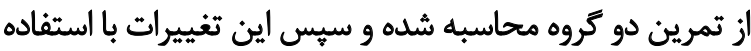

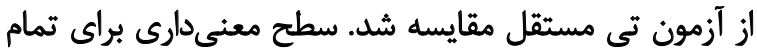

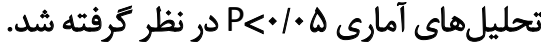

يافتهها

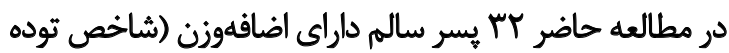

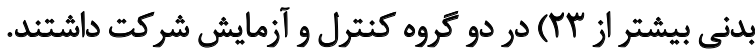
ميانكين سنى در كروه آزمايش

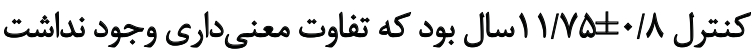

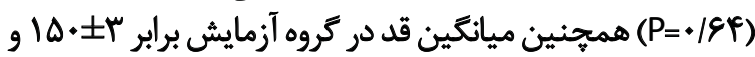

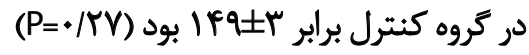

دادهاى وزن، شاخص توده بدنى، درصد جربي،، نسبت دوركمر

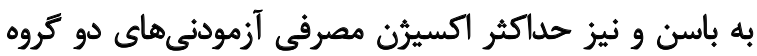

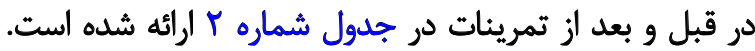

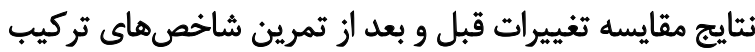

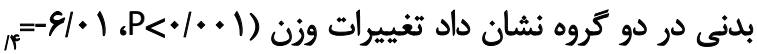

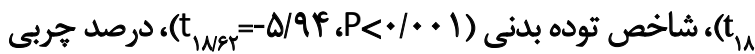

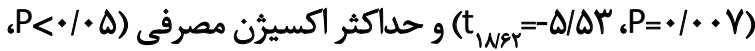
Sرو

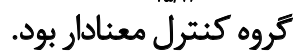
تحليل آمارى دادهها تفاوت معنادارى را بين تغييرات سطوح

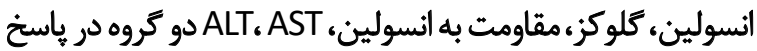

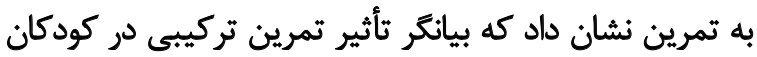

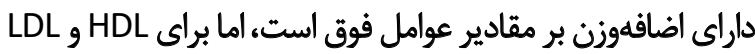
تفاوت معنى دارى مشاهده نشد (جدول شماره باد).

\section{ث}

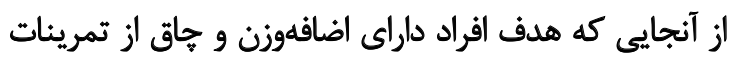

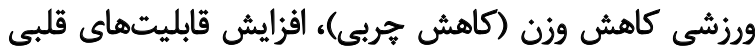

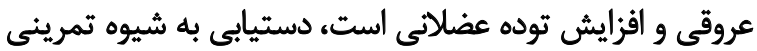

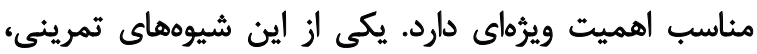

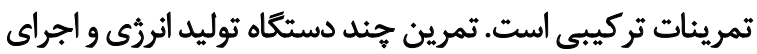

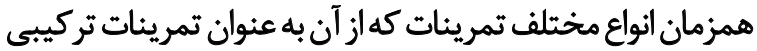

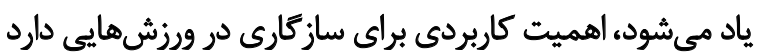

تمرينات هوازى و مقاومتى را انجام دادند (جدول شماره (). از

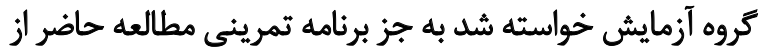

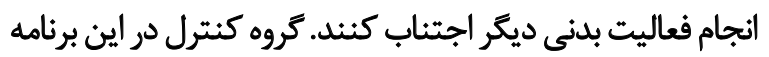

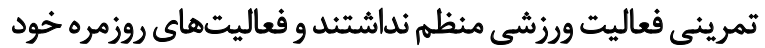

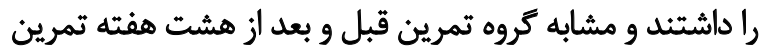

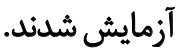

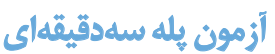
در ابتداي مطالعه و در انتهاي هشت هفته از همه كودكان در

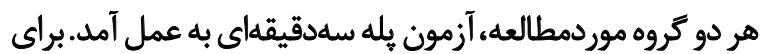

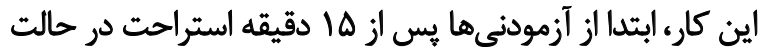

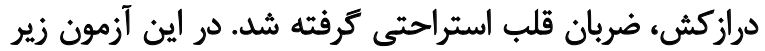

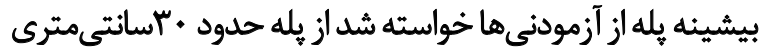

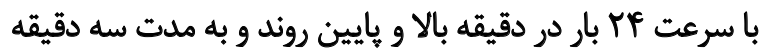

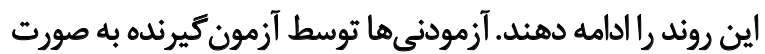

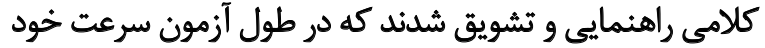

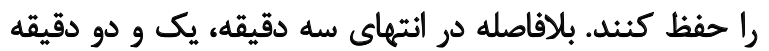

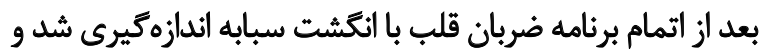

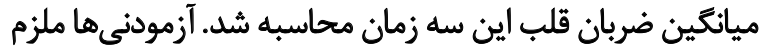

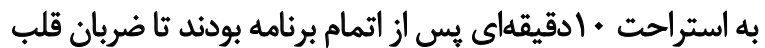

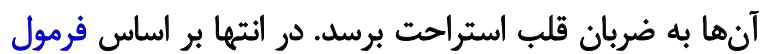

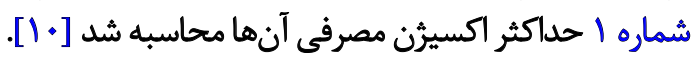
فرمول شماره 1. × (قد بر حسب سانتىمتر

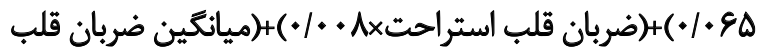

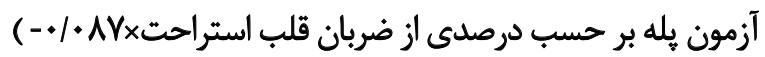

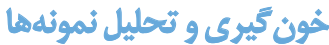

در ابتداى مطالعه و در انتهاى هشت هفته، از همه آزمودنى ها ها

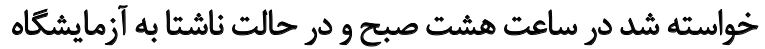

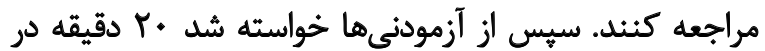

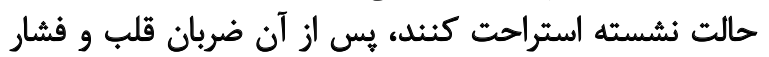

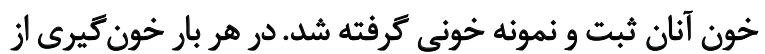

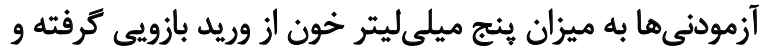

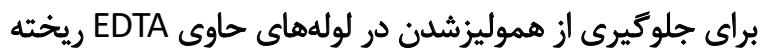

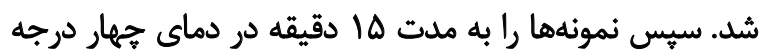

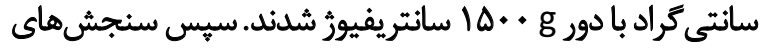

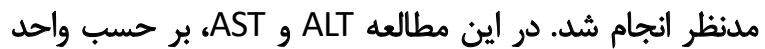

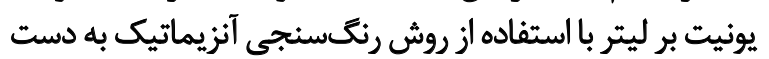

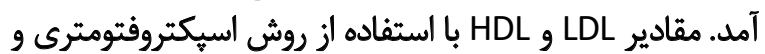

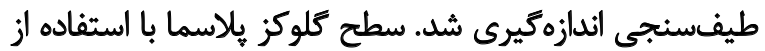

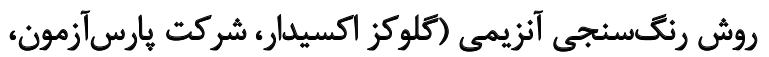

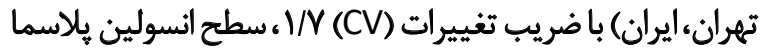


جدول r. دادههاي تركيب بدنى دو كروه يسران سالمم با اضافهوزن در ابتدا و پايان مطالعه (اهنفر)

\begin{tabular}{|c|c|c|c|c|}
\hline \multicolumn{4}{|c|}{ ميانعين +انحراف معيار } & \multirow{3}{*}{ زمان } \\
\hline \multicolumn{2}{|c|}{ كروه كتترل } & \multicolumn{2}{|c|}{ كروه آزمايش (تمرين تركيبى) } & \\
\hline نايان هفته هشتم & 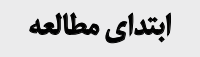 & بايايان هفته هشتم & ابتداى مطالعه & \\
\hline$\Delta \cdot / f \pm Q / V$ & $r q / \Delta \pm N r$ & $P e / \Delta \pm \& / r$ & $\Delta \cdot / \varepsilon \pm V / A$ & و ونْن (كيلوكرم) \\
\hline$r A / Y \pm N A$ & $r \Delta / r \pm T / r$ & $\pi / r \pm M$ & $r \Delta / \uparrow \pm Y /$. & شاخص توده بلدى (كيلوكرم / متر مريع") \\
\hline$r F / r \pm q \Delta / \Delta \Lambda$ & $M \in / N \pm r V / A r$ & $r M / r \pm 8 / / 9 P$ & $r f / \Delta \pm I V / 1 \Delta$ & درصد هربى (درصد) \\
\hline $.1 . \pm 91 / 11$ & $.1 . \pm 91 / .9$ & $\cdot 1 \cdot \pm 9 \cdot 1 \cdot 1$ & $\cdot 1 \cdot \pm q r / 10$ & (نسبت) WHR \\
\hline YIAV/IMTI $\pm \Delta \cdot / I V$ & MIQSITrET/QS & $M T q P / M I \Delta \pm R T / M P$ & MUARLEITE/M. & حلاكثر اكسيرثن مصرفى ( ليتر / دقيقه) \\
\hline
\end{tabular}

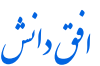

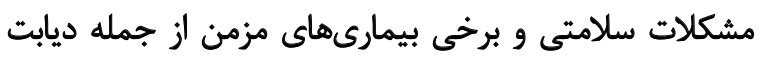

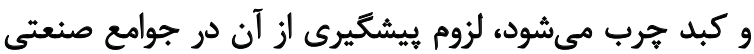

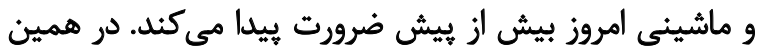

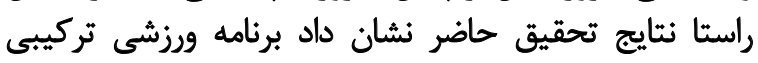

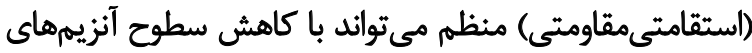

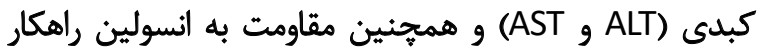

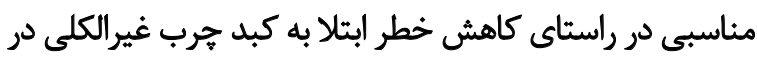

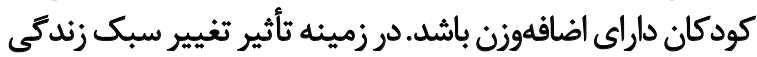

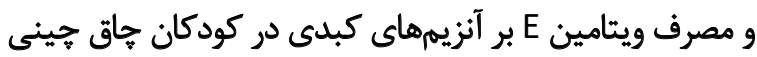

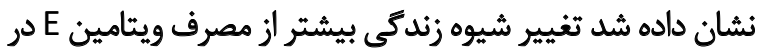

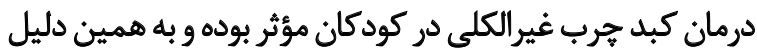

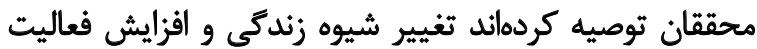

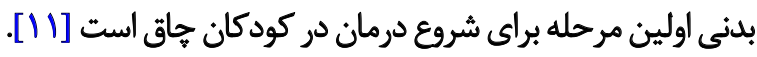

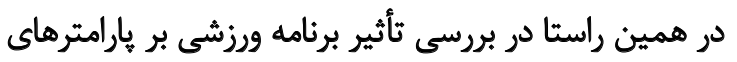

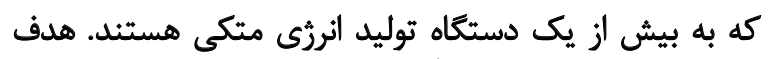

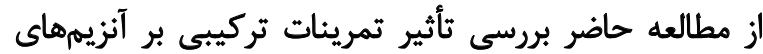

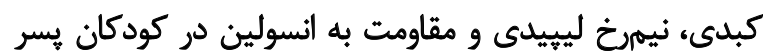

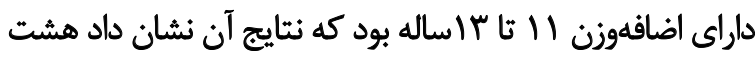

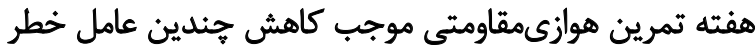

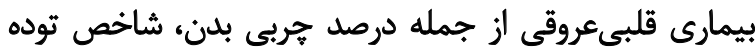

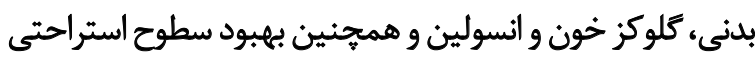

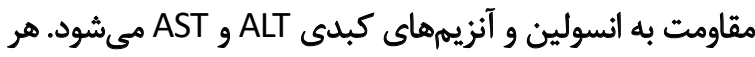

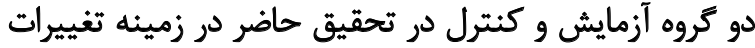

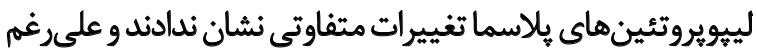

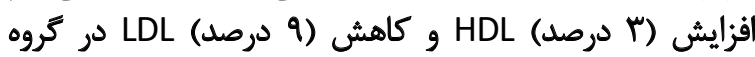

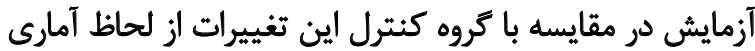

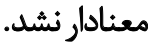
با توجه به شيوع جاقى در ميان كودكان كه خود زمينهساز

جدول ז. مقايسه تغييرات دادهماي دو كروه بسران سالم با اضافهوزن در ابتدا و بايان مطالعه با آزمون تى مستقل (اهنفر)

\begin{tabular}{|c|c|c|c|c|c|}
\hline \multirow{3}{*}{ Pقدار P P P } & \multicolumn{4}{|c|}{ ميانكين ثانحراف معيار } & \multirow{3}{*}{ 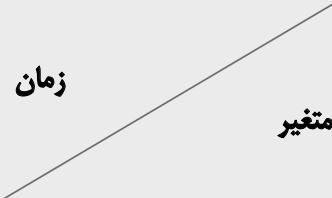 } \\
\hline & \multicolumn{2}{|c|}{ كروه كتترل } & \multicolumn{2}{|c|}{ كروه أزمايش (تمرين تركيبى) } & \\
\hline & يايان هفته هشتم & ابتداى مطالعه & يايايان هفته هشتم & ابتداى مطالعه & \\
\hline $\mathrm{P}=+(*)^{*}$ & $\mid r / ৭ q \pm \Psi / \| f$ & $q V / r q \pm I r / r$ & $1 \cdot / N \Delta \pm \Psi / N E$ & $1 r / N \Delta \pm r / N^{\prime}$ & انسولين (ميكرويونيت بر ميلى ليتر) \\
\hline $\mathrm{P}=* 1.11^{*}$ & $M W \Psi \varepsilon / F$ & $\triangle \vee / W \pm \Delta / Y$ & $\Lambda Q / f T \pm V / \&$ & $9 \varphi / \& \pm V / \Lambda$ & كلوكز ميلى كرم بر دسى ليتر \\
\hline $\mathrm{P}=* \mid *)^{*}$ & $r / \cdot V \pm \cdot M$ & T/AY士./8Q & $r / T r E \pm . / Q E$ & $M / M E \pm . N A$ & مقاومت به انسولين \\
\hline $\mathrm{P}=.(\cdot .)^{*}$ & $r / r \pm E q / Q$ & $M / A \Delta \pm r / W$ & RV/AEIY/YG & $M / \Delta I \pm T / T$. & ALT يونيت بر ليتر \\
\hline $\mathrm{P}=* / *)^{*}$ & $r V / r \pm+\Delta / A Y$ & $r e / E q \pm r / \cdot r$ & $r / R+A \Delta / \cdot r$ & $r E / \Delta F \pm \Delta / F q$ & AST يونيت بر ليتر AST \\
\hline $\mathrm{P}=. / 9 \vartheta$ & $P V / r V \pm V / W$ & $P \Delta / A \pm q / \Delta T$ & $\Delta \cdot / M \pm E / P \mid$ & $F N V \cdot \pm r / M$ & HDL ميلى كرم بر دسىليتر \\
\hline $\mathrm{P}=. / 11 \mathrm{~V}$ & QF/QY $\pm \mid \& / A F$ & $1 .+/ 11 \pm V / \Delta q$ & $N / R \pm 19 / M P$ & $9 . / 1 \pm r / \Delta F$ & ميلى كرم بر دسى ليتر LDL \\
\hline
\end{tabular}




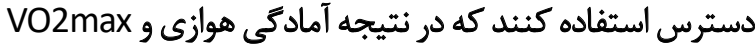

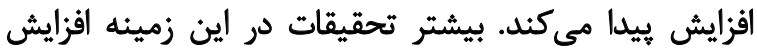

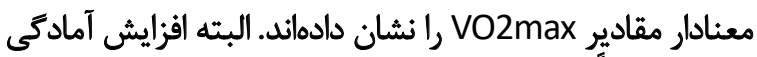

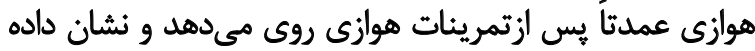

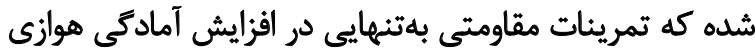

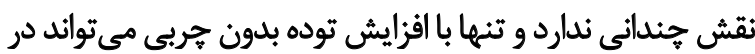

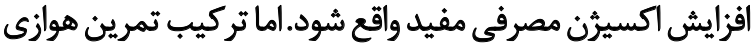

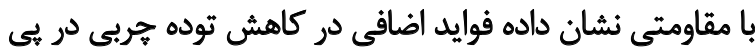

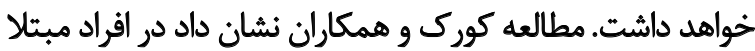

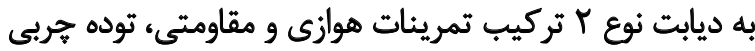

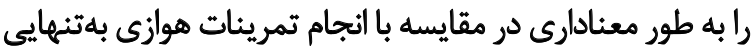

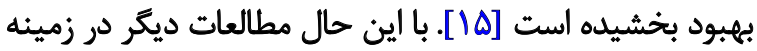

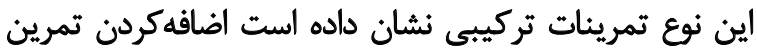

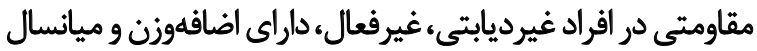

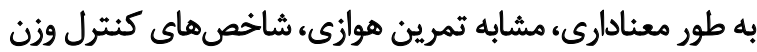
رادجار تغيير كرده است [19]".

نتايج متناقض اين مطالعات ناشى از اين موضوع است كه

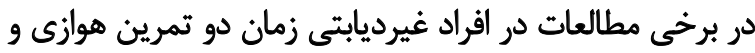

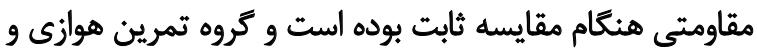

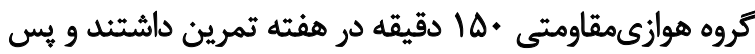

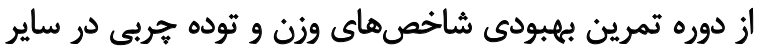

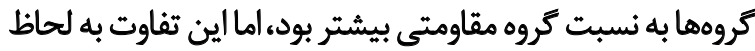

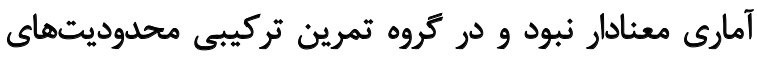

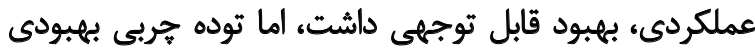

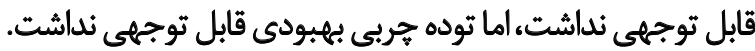

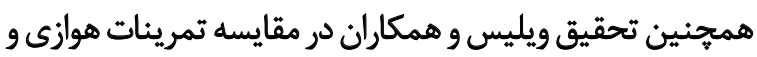

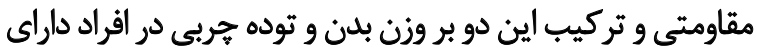

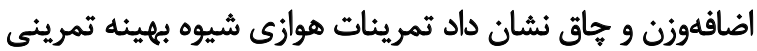

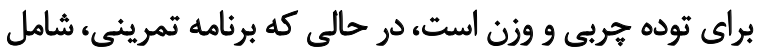

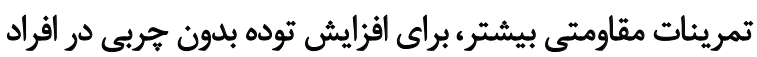

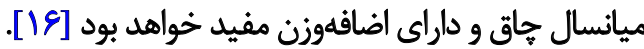

علاوه بر وزن و درصد جربى بدن، شواهد زيادى نيز نشان

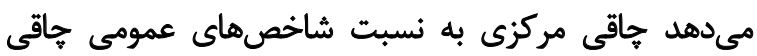

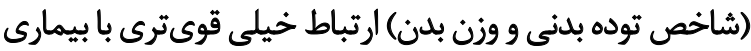

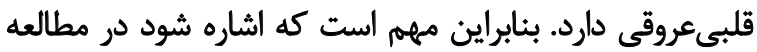

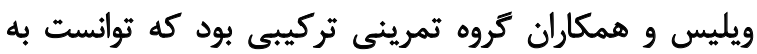

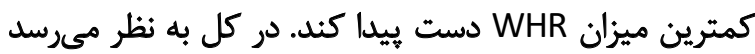

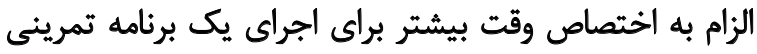

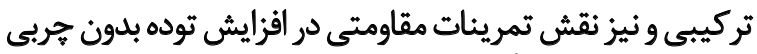

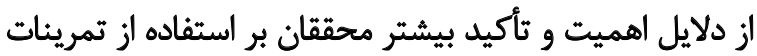

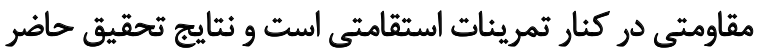

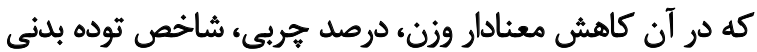

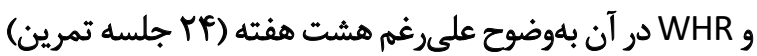
مشاهده شد، نشاندهنده الهميت و تأثير تذارى ائن اين نوع تمرينات تمرين
قلبى عروقى، متابوليكى و آنترويومتريكى در كودكان جاق نشان

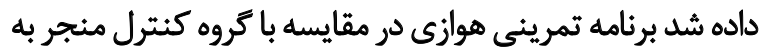

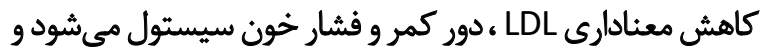

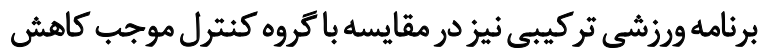

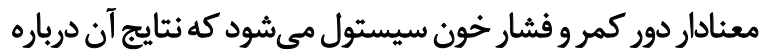

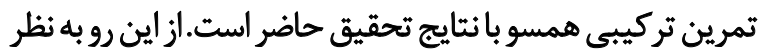

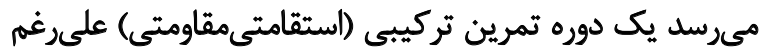

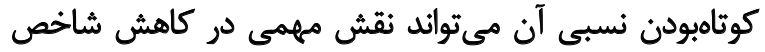

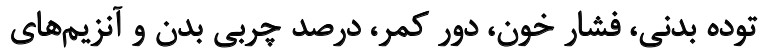

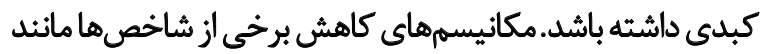

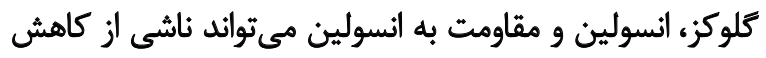

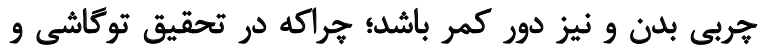

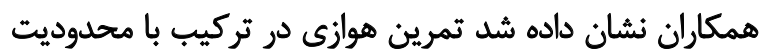

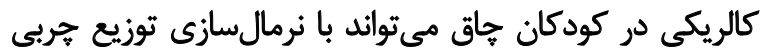

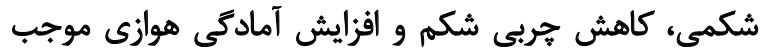

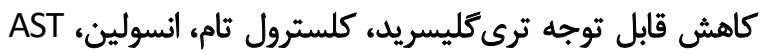

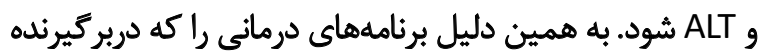

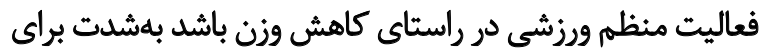

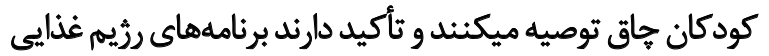

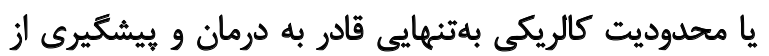
آسيبهاى جاقى در كودكان نيست [IIT)]

تمرين ورزشى منظم، ميزان لييوليز رادر بافت جربيى افزايش

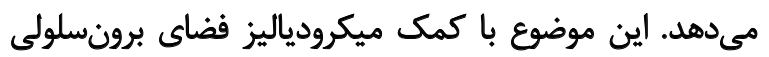

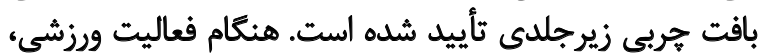

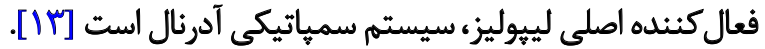

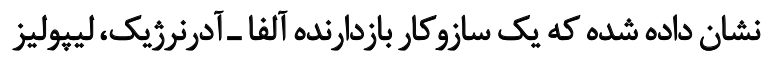

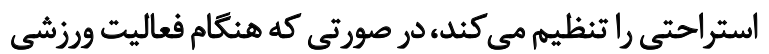

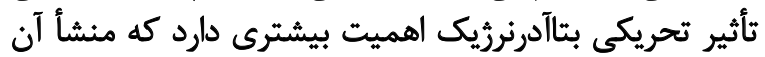

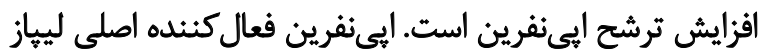

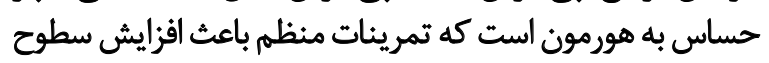

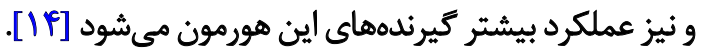
در تحقيق حاضر WHR بس از هشت هفته تمرين تركيبى در

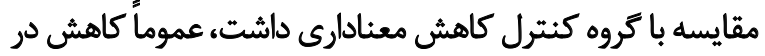

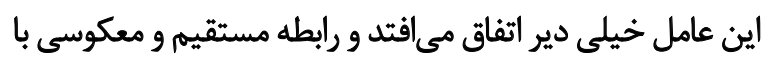

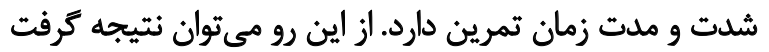

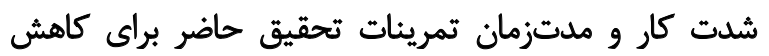

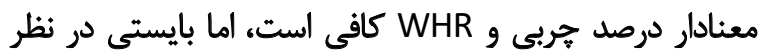

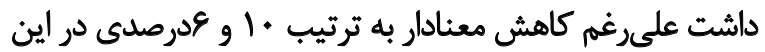

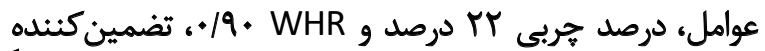
در معرض خطر نبودن اين كودكان نيست و و براي اينكه كامداملاً

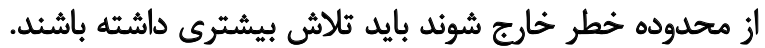

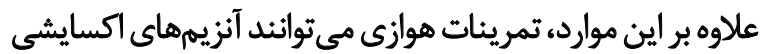

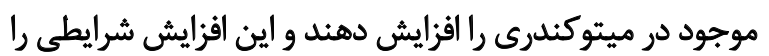

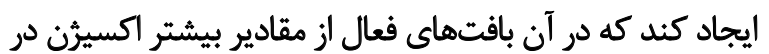




\section{نتيجهيَيرى}

با توجه به يافتههاي مطالعه حاضر، تمرينات تركيبى (هوازي كاري

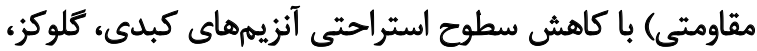

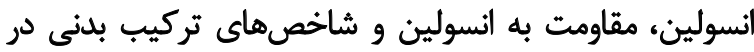

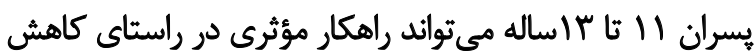

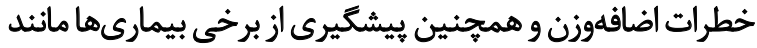

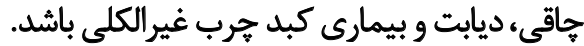

بايستى تأكيد كرد علىرغم كاهش معنادار بسيارى از باز

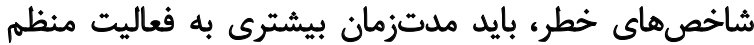

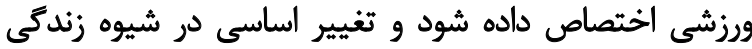

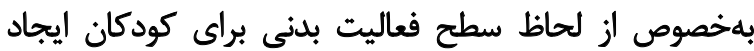

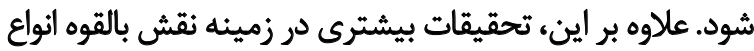

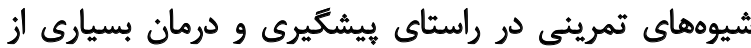

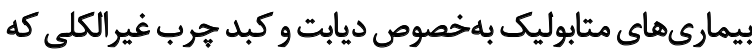
در ميان كودكان شيوع بالايى بيدا كرده لازم است.

مطالعه حاضر محدوديتهايى داشت از جمله اينكه امكان

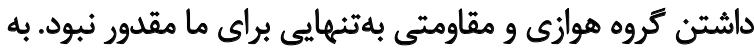

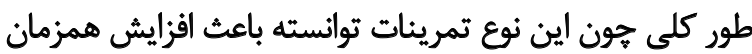

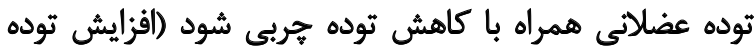

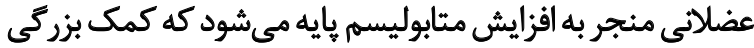

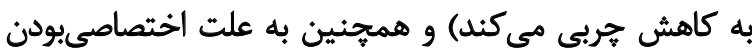

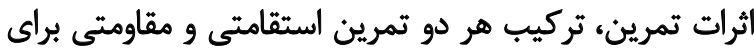

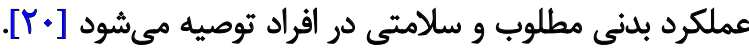

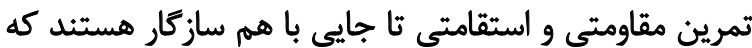
تعداد جلسات تمرين استقامتى كم نشودي

مالاحظات اخلاقى

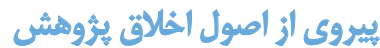

با توضيح اهداف و جزئيات مطالعه، رضايتنامه كتبى و آكاهانه

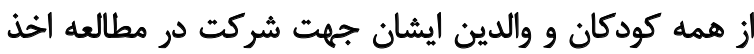

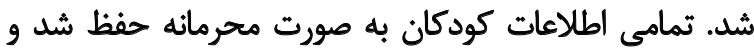

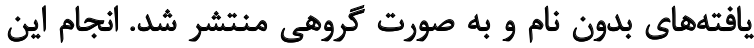

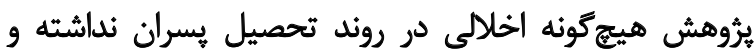

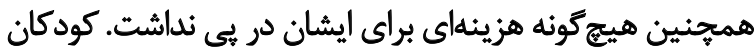

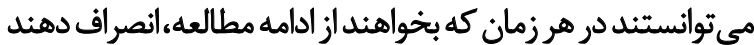

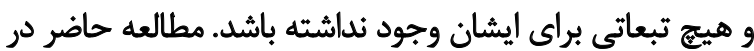

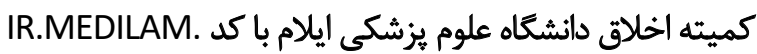
REC.1397.030 تصويب شده است.

$$
\text { إمب }
$$

اين مطالعه با هزينه شخصى نويسندكان انجام شده است.
در كودكان داراى اضافهوزن در راستاى بيشكَيرى و در درمان

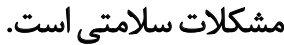

بالابودن مقاومت به انسولين افراد جاق و داراى اضافهوزن در

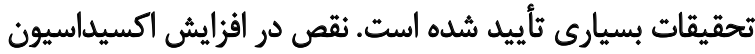

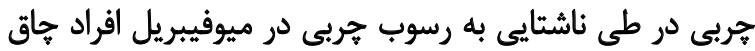

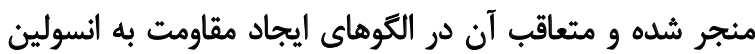

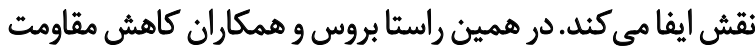

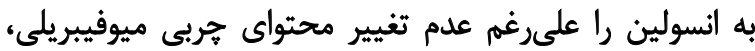

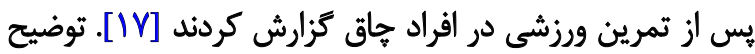

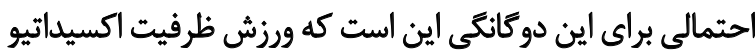

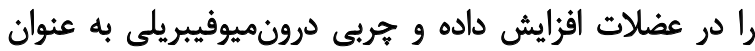

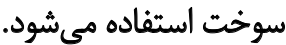

محققان بسيارى اثر ورزش منظم را بر مقاومت به به انسولين بودين

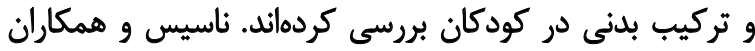

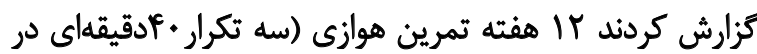

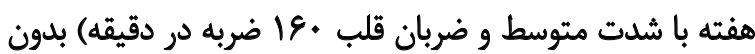

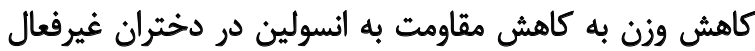

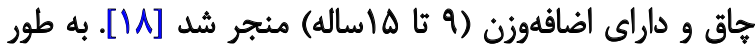

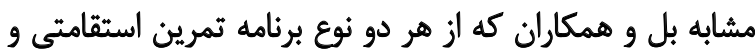

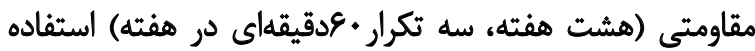

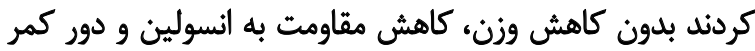

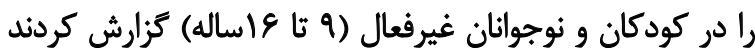

اكرجه هر دو مطالعهاي كه به آنها اشاره شد از نظر جنسيت

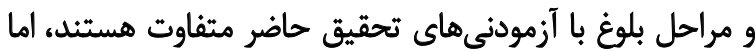

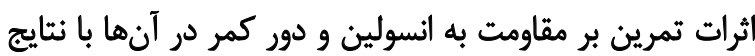

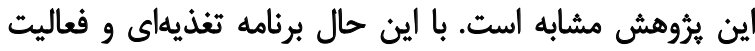

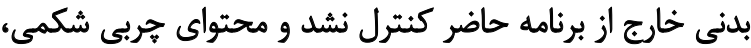
كبدى و درونميوفيبريلى نيز اندازمكيرى نشد.

در تحقيق هيدن و همكاران انسولين ناشتايى ارتباط بسيار قوى

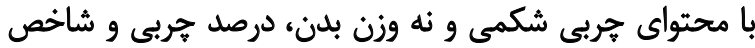

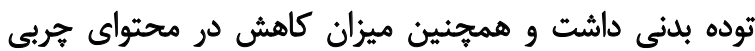

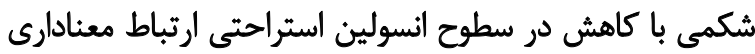

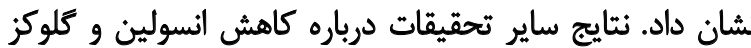

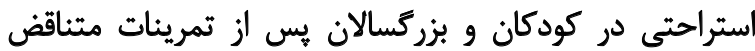

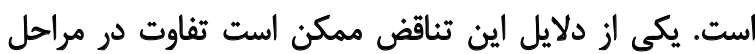

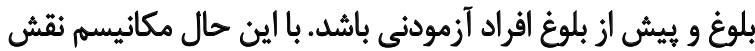

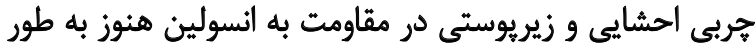

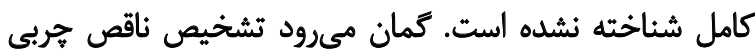

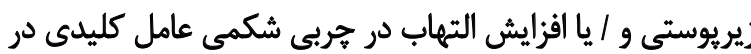

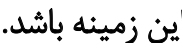


مشار كت نويسند مكان

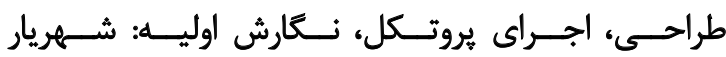

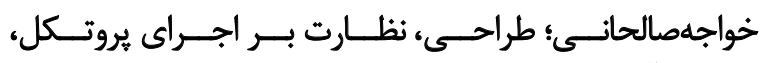

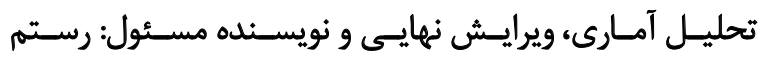
علــيزاده. تعارض مثافع

نويسندكان تصريح مى كنئد كه هيج مقاله وجود ندارد. 


\section{References}

[1] Watts K, Jones TW, Davis EA, Green D. Exercise training in obese children and adolescents. Sports Medicine. 2005; 35(5):375-92. [DOI:10.2165/00007256-200535050-00002] [PMID]

[2] Watts K, Beye P, Siafarikas A, Davis EA, Jones TW, O'Driscoll G, et al. Exercise training normalizes vascular dysfunction and improves central adiposity in obese adolescents. Journal of the American College of Cardiology. 2004; 43(10):1823-7. [DOI:10.1016/j.jacc.2004.01.032] [PMID]

[3] Kim ES, Im JA, Kim KC, Park JH, Suh SH, Kang ES, et al. Improved insulin sensitivity and adiponectin level after exercise training in obese Korean youth. Obesity. 2007; 15(12):3023-30. [DOI:10.1038/ oby.2007.360] [PMID]

[4] Woo KS, Chook P, Yu CW, Sung RY, Qiao M, Leung SS, et al. Effects of diet and exercise on obesity-related vascular dysfunction in children. Circulation. 2004; 109(16):1981-6. [DOI:10.1161/01. CIR.0000126599.47470.BE] [PMID]

[5] Ferguson MA, Gutin B, Le NA, Karp W, Litaker M, Humphries M, et al. Effects of exercise training and its cessation on components of the insulin resistance syndrome in obese children. International Journal of Obesity. 1999; 23(8):889-95. [DOI:10.1038/sj.ijo.0800968] [PMID]

[6] Marques EA, Mota J, Viana JL, Tuna D, Figueiredo P, Guimarães JT, et al. Response of bone mineral density, inflammatory cytokines, and biochemical bone markers to a 32-week combined loading exercise programme in older men and women. Archives of Gerontology and Geriatrics. 2013; 57(2):226-33. [DOI:10.1016/j.archger.2013.03.014] [PMID]

[7] Blimkie CJ. Resistance training during preadolescence. Sports Medicine. 1993; 15(6):389-407. [DOI:10.2165/00007256-19931506000004] [PMID]

[8] Oh S, Shida T, Sawai A, Maruyama T, Eguchi K, Isobe T, et al. Acceleration training for managing nonalcoholic fatty liver disease: A pilot study. Therapeutics and Clinical Risk Management. 2014; 10:925-36. [DOI:10.2147/TCRM.S68322] [PMID] [PMCID]

[9] Dill DB, Costill DL. Calculation of percentage changes in volumes of blood, plasma, and red cells in dehydration. Journal of Applied Physiology. 1974; 37(2):247-8. [DOI:10.1152/jappl.1974.37.2.247] [PMID]

[10] Jacks DE, Topp R, Moore JB. Prediction of VO2 peak using submaximum bench step test in children. Clinical Kinesiology. 2012; 66(3):74-81.

[11] Wang CL, Liang L, Fu JF, Zou CC, Hong F, Xue JZ, et al. Effect of lifestyle intervention on non-alcoholic fatty liver disease in Chinese obese children. World Journal of Gastroenterology. 2008; 14(10):1598-602. [DOI:10.3748/wjg.14.1598] [PMID] [PMCID]

[12] Togashi K, Masuda H, Iguchi K. Effect of diet and exercise treatment for obese Japanese children on abdominal fat distribution. Research in Sports Medicine. 2010; 18(1):62-70. [DOI:10.1080/15438620903423924] [PMID]

[13] Zemel MB, Thompson W, Milstead A, Morris K, Campbell P. Calcium and dairy acceleration of weight and fat loss during esnergy restriction in obese adults. Obesity Research. 2004; 12(4):582-90. [DOI:10.1038/oby.2004.67] [PMID]

[14] Nicklas BJ, Rogus EM, Goldberg AP. Exercise blunts declines in lipolysis and fat oxidation after dietary-induced weight loss in obese older women. American Journal of Physiology-Endocrinology and Metabolism. 1997; 273(1):E149-55. [DOI:10.1152/ajpendo.1997.273.1.E149] [PMID]
[15] Church TS, Blair SN, Cocreham S, Johannsen N, Johnson W, Kramer K, et al. Effects of aerobic and resistance training on hemoglobin A1c levels in patients with type 2 diabetes: A randomized controlled trial. JAMA. 2010; 304(20):2253-62. [DOI:10.1001/ jama.2010.1710] [PMID] [PMCID]

[16] Willis LH, Slentz CA, Bateman LA, Shields AT, Piner LW, Bales CW, et al. Effects of aerobic and/or resistance training on body mass and fat mass in overweight or obese adults. Journal of Applied Physiology. 2012; 113(12):1831-7. [DOI:10.1152/japplphysiol.01370.2011] [PMID] [PMCID]

[17] Bruce CR, Thrush AB, Mertz VA, Bezaire V, Chabowski A, Heigenhauser GJ, et al. Endurance training in obese humans improves glucose tolerance and mitochondrial fatty acid oxidation and alters muscle lipid content. American Journal of Physiology-Endocrinology and Metabolism. 2006; 291(1):E99-107. [DOI:10.1152/ajpendo.00587.2005] [PMID]

[18] Nassis GP, Papantakou K, Skenderi K, Triandafillopoulou M, Kavouras $\mathrm{SA}$, Yannakoulia $\mathrm{M}$, et al. Aerobic exercise training improves insulin sensitivity without changes in body weight, body fat, adiponectin, and inflammatory markers in overweight and obese girls. Metabo lism. 2005; 54(11):1472-9. [DOI:10.1016/j.metabol.2005.05.013] [PMID]

[19] Bell LM, Watts K, Siafarikas A, Thompson A, Ratnam N, Bulsara M, et al. Exercise alone reduces insulin resistance in obese children independently of changes in body composition. The Journal of Clinical Endocrinology \& Metabolism. 2007; 92(11):4230-5. [DOI:10.1210/ jc.2007-0779] [PMID]

[20] Coffey VG, Hawley JA. The molecular bases of training adaptation. Sports Medicine. 2007; 37(9):737-63. [DOI:10.2165/00007256200737090-00001] [PMID] 
This Page Intentionally Left Blank 